\title{
THE NAVAL OBSERVATORY "SHORT" PLATE BEING TAKEN AS PART OF THE PALOMAR SKY SURVEY II
}

\author{
David G. Monet \\ U. S. Naval Observatory Flagstaff Station \\ P. O. Box 1149, Flagstaff, AZ 86002, USA
}

\begin{abstract}
The US Naval Observatory has commissioned a short exposure plate for each field in the Palomar Sky Survey II. These plates should provide the necessary intermediate reference frame for the determination of right ascension and declination for faint objects on the "deep" plates using catalog coordinates for bright stars measured on the "short" plate.
\end{abstract}

\section{INTRODUCTION}

The US Naval Observatory (USNO) and the Flagstaff Station (NOFS) have initiated a collaboration with the California Institute of Technology for the measurement of all plates taken for the first and second Palomar Sky Surveys (PSS-I and PSS-II). Of particular importance to the USNO mission is the determination of right ascensions, declinations, proper motions, magnitudes, and colors for large numbers of faint stars. An engine capable of measuring and reducing this large amount of plate material has been designed. A prototype engine was constructed at the Kitt Peak National Observatory (KPNO) of the National Optical Astronomy Observatory, and is available as a user facility. The building to house the production measuring engine has been built at NOFS.

Preparations for this measuring program fall into four major areas. First, the requirements for the "short" exposure plates must be determined. Second, a measuring engine with a bandwidth of order 60 microseconds of time per square arcsecond of sky is needed to generate the internal measures. Third, astrometric and photometric calibrations must be applied to convert the internal measures to a useful external system. Finally, list correlation software is needed to merge the star lists and compute proper motions. This discussion centers on the first and second areas.

\section{THE USNO "SHORT" PSS-II PLATE}

As is well known, virtually all stars with well determined coordinates (AGK3R, SAO, etc.) are hopelessly overexposed on the deep PSS-I and PSS-II plates. This problem was avoided by the Guide Star Selection System (GSSS) for the Space Telescope (Russell 1984, and references cited therein). Their "Quick V" survey exposure was short enough so that many stars with known coordinates 
could be measured while still attaining a faint limit appropriate for Space Telescope pointing. As with all compromises, the GSSS plates are of limited use. They used IIa-D emulsions, a 20 minute exposure, and measured with a 25 micron aperture. GSSS catalog stars will rarely be fainter than 16th magnitude, and the typical epoch of observation is early 1984.

In order to get astrometric information for many more stars and other objects, the USNO decided to commission a "short" PSS-II survey plate. This will be taken in addition to the $J, F$, and $N$ plates originally scheduled. The following list contains most of the important characteristics of the "short" survey plates.

- IIIa-J emulsion, no hypersensitization, GG385 filter, and a 3 minute nominal exposure.

- The "short" and "deep" $J$ plates will probably be taken in the same dark run, but probably not on the same night. The USNO has suggested a limit of no more than \pm 1 month epoch difference.

- The "short" plates will be subjected to the same seeing and hour angle constraints as regular PSS-II plates.

- The "short" plates should be taken with the same plate holder and filter as the "deep" plates, but this cannot be guaranteed.

- The plates remain the property of the USNO but will be stored with the PSS-II plates and copies will be made available in media and cost similar to regular PSS-II plates.

Several test plate sequences were taken at Palomar and measured at KPNO. While these plates were not acceptable for PSS-II, they did provide some quantitative results. In a $2 \times 2$ degree medium latitude field, more than 30 AGK3R stars were measurable on the "short" plate and more than 500 stars were measured on both the "short" and "deep" plates. While these tests are not comprehensive, they do show that the intermediate astrometric reference frame has enough stars to define the mapping from the AGK3R coordinate system to the faint limit of the PSS-II.

\section{STATUS OF THE KPNO PROTOTYPE ENGINE}

As mentioned previously, a prototype of the measuring engine was constructed early in 1984 based on the KPNO PDS measuring engine, a VAX 11/750, an IIS Model 70 display device, and a Fairchild CCD221. This prototype serves two purposes. First, scientific results are being produced (Saha et.al. 1987). Second, the image processing software is being developed and refined. This gives the first tangible evidence that the data acquisition and processing rate meets the system requirement of one sky per year.

Perhaps the weakest link of the prototype system is the CCD television camera. This device uses line transfer technology so it does not have square pixels, a square lattice, or one pixel per digitized RS-170 video format pixel. Fortunately, the prototype system is in the process of being upgraded and improved. The current plan is to install a GE TN2250 CID camera. This device has square, contiguous 15-micron pixels, a $512 \times 512$ array, and can be slow scanned directly into the A/D converter of the frame grabber. RS-170 video format is avoided while still retaining a $10-\mathrm{Hz}$ frame rate and well defined pixels. The cost of this camera and frame grabber system is about $\$ 6000$. In addition, a MicroVAX II will replace the overloaded host VAX 11/750 speeding up both the measuring engine and the KPNO VMS IRAF user programs. 


\section{REFERENCES}

Russell, J.L. 1984, in: IAU Symposium 109: Astrometric Techniques, ed. H.K. Eichhorn and R.J. Leacock, (Dordrecht:Reidel), p721.

Saha, A., Monet, D., and Seitzer, P. 1986, Astron.J., 92, 302. 